Check for updates

Cite this: RSC Adv., 2019, 9, 8290

Received 26th December 2018 Accepted 25th February 2019

DOI: $10.1039 / \mathrm{c} 8 \mathrm{ra10570g}$

rsc.li/rsc-advances

\section{One-step hydrothermal synthesis and optical properties of self-quenching-resistant carbon dots towards fluorescent ink and as nanosensors for $\mathrm{Fe}^{3+}$ detection}

\begin{abstract}
Dandan Xu, ${ }^{a}$ Fang Lei, (D) *a Haohong Chen, ${ }^{\mathrm{b}}$ Luqiao Yin, ${ }^{\mathrm{c}}$ Ying Shi*a and Jianjun Xie ${ }^{\mathrm{a}}$
In our work, blue photoluminescent $\mathrm{N}$-doped carbon dots (CDs) were developed via a green and simple hydrothermal method with citric acid and polyvinyl pyrrolidone (PVP K-30) as the carbon source and the nitrogen source, respectively. The as-prepared CDs have a high fluorescent quantum yield of $30.21 \%$ and considerable luminescence stability. The fluorescence intensity of the CDs was found to be effective quenched when adding $\mathrm{Fe}^{3+}$ ions to the $\mathrm{CDs}$ solution. The quenching phenomenon can be used to

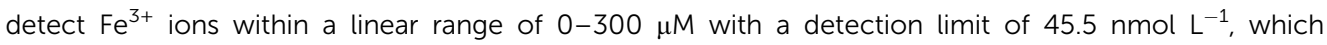
suggested its potential application in the detection of $\mathrm{Fe}^{3+}$ ions. At the same time, we also noted the excellent self-quenching-resistant property of the as-prepared CDs in the solid state, and bright blue fluorescence was observed under UV excitation. What's more, the as-prepared CDs can also be used as fluorescent ink and were presented under UV excitation.
\end{abstract}

\section{Introduction}

In the past few years, as a new kind of carbon materials, carbon dots (CDs) have great prospects in the application of luminescence, biomedical and the ion detection filed. CDs usually refer to a new class of carbon nanomaterials with a size below $10 \mathrm{~nm}$, which were firstly founded by $\mathrm{Xu}$ through the purification of single-walled carbon nanotubes in $2004 .^{1}$ As the new member of the carbon nanomaterials family, CDs are comparable or even alternative materials to traditional semiconductor quantum dots (QDs), such as CdSe, PbS, and CdS, etc., which are widely used in bioimaging and biosensing, ${ }^{2-7}$ patterning, ${ }^{8,9}$ coding, ${ }^{10}$ and optoelectronics ${ }^{11-13}$ for their water solubility, good stability, low toxicity, superior resistance to photobleaching, and excellent biocompatibility. ${ }^{\mathbf{1 4 1 5}}$ The wide application of CDs mainly comes from their luminescent properties. Although the PL mechanism of CDs is controversial, more and more evidence shows that the (photoluminescence) PL emission of CDs is due to the synergistic effect of the non-intrinsic PL of the CDs core and surface state. In addition, a large number of studies have shown that heteroatom doping can significantly tune their electronic properties, surface and local chemistry, as well as

${ }^{a}$ School of Materials Science and Engineering, Shanghai University, 99 Shang Da Road, Shanghai 200444, PR China.E-mail:leif@shu.edu.cn; yshi@shu.edu.cn

${ }^{b}$ Key Laboratory of Transparent Opto-functional Inorganic Materials, Shanghai Institute of Ceramics, Chinese Academy of Sciences, Dingxi Road 1295, Shanghai 200050, China

${ }^{c}$ Key Laboratory of Advanced Display and System Applications, Shanghai University, 149 Yanchang Road, Shanghai 200072, P. R. China extend their applications. Currently, heteroatoms like nitrogen, phosphorus, sulphur, boron and fluorine have been confirmed to improve the PL quantum yield and PL intensity of the carbon dots. The doping of the heteroatom can introduce holes or electrons to the carbon dots, which distinctly improve the electronic structure and chemical activity, thus improving the optical and electrical properties for a more extensive application. Due to the $\mathrm{N}$ atom having a similar atomic radius to the $\mathrm{C}$ atom and the outer five valence electrons to bind carbon atoms, doping with nitrogen is widely used. ${ }^{16}$ For example, Wang et al. reported a kind of $\mathrm{N}$-CDs synthesised by the hydrothermal method from streptomycin, which was used as fluorescent probes in cell imaging. ${ }^{17}$ Lu et al. used a mixture of oxalic acid and urea as a precursor to synthesize blue fluorescent $\mathrm{N}$-doped carbon dots, which were used to detect $\mathrm{Fe}^{3+}$ and $\mathrm{Ag}^{+} \cdot{ }^{18}$ Dong et al. used a mixture of citric and branched polyethyleneimines as a precursor for the one-step carbonization synthesis of carbon dots and used them for chemical sensing. ${ }^{19}$ However, the low-fluorescence quantum yield and self-quenching of solid-state CDs can be ascribed to excessive resonance energy transfer (RET) or the direct $\pi-\pi$ interactions of organic molecules, which seriously limit the development of CDs. ${ }^{20}$

To our knowledge, there are few reports about the one-step preparation of CDs with solid-state luminescence. ${ }^{21,22}$ What's more, reports that mentioned the solid-state fluorescence (SSF) of CDs usually require the recombination of CDs and a solid matrix such as a polymer matrix to obtain the SSF. However, CDs as polymer materials generally have a fixed shape, which are inconvenient to apply in many fields compared with 
phosphors. Therefore, there is an urgent need to further research CDs-based phosphors with self-quenching-resistant property using environmentally friendly and widely available raw materials through a simple, low-cost, convenient method.

As we all know, $\mathrm{Fe}^{3+}$ ions, as one of the most essential metal ions in biological systems, plays a crucial role in the human body and the environment. ${ }^{23}$ However, excess amounts of $\mathrm{Fe}^{3+}$ ions in the human body and environment may cause some diseases and serious environmental pollution. In the past few years, many chemosensors based on organic dyes, ${ }^{24}$ semiconductor quantum dots, ${ }^{25}$ metal nanoparticles,${ }^{26}$ and fluorescent meal organic frameworks ${ }^{27}$ have been widely used as a sensing probe of $\mathrm{Fe}^{3+}$. However, most chemosensors are poisonous, biologically incompatible and water-insoluble, which greatly limited their application in detection of $\mathrm{Fe}^{3+}$. So, the development of a new fluorescent $\mathrm{Fe}^{3+}$ sensing probe with photostability, high sensitivity and selectivity that permits naked-eye detection is truly meaningful. Carbon dots (CDs) have the advantages of water-solubility, low toxicity, good biocompatibility, and chemical stability. ${ }^{28,29}$ Additionally, $\mathrm{Fe}^{3+}$ could induce the photoluminescence quenching of carbon dots by the special coordinate interaction between $\mathrm{Fe}^{3+}$ and the surface groups of carbon dots. These phenomena enabled carbon dots to act as a nanosensor for $\mathrm{Fe}^{3+}$ detection.

In this paper, we prepared blue-emission N-CDs with a fluorescent quantum yield of $30.21 \%$ via a one-step hydrothermal method using citric acid and polyvinyl pyrrolidone (PVP K-30) as the carbon source and the nitrogen source. Citric acid and polyvinyl pyrrolidone (PVP K-30) are low-cost, widely available and environmentally friendly materials with excellent performance. Compared with previous reports, ${ }^{4,30}$ our method avoids using complicated raw materials and surface passivation, which is convenient for industrial production. During the experimental process, we found that the as-prepared CDs show great potential in the field of the detection of $\mathrm{Fe}^{3+}$ ions. There was a linear correlation between fluorescent intensity and the concentration of $\mathrm{Fe}^{3+}$ with a linear range from $0-300 \mu \mathrm{M}$ and a detection limit of $45.5 \mathrm{nmol} \mathrm{L}^{-1}$, which indicates that the detection sensitivity in our work is higher than the limit of detection (LOD) data reported before. ${ }^{31,32}$ Furthermore, the asprepared CDs demonstrate excellent blue fluorescence in the solid state after freeze-drying to a powder, which provides more possibilities for the application of CDs in ion detection and solid-state lighting devices. Additionally, the CDs can also be used as a fluorescent ink and are presented under UV excitation.

\section{Experimental}

\section{Chemicals and materials}

Citric acid (CA), polyvinyl pyrrolidone (PVP K-30), acetone, $\mathrm{Cu}\left(\mathrm{NO}_{3}\right)_{2}, \mathrm{MnCl}_{2} \cdot 4 \mathrm{H}_{2} \mathrm{O}, \mathrm{CaCl}_{2}, \mathrm{ZnCl}_{2}, \mathrm{FeSO}_{4} \cdot 7 \mathrm{H}_{2} \mathrm{O}, \mathrm{Fe}\left(\mathrm{NO}_{3}\right)_{3}-$ $\cdot 9 \mathrm{H}_{2} \mathrm{O}, \mathrm{NaCl}, \mathrm{MgCl}_{2}, \mathrm{Ni}\left(\mathrm{NO}_{3}\right)_{2} \cdot 6 \mathrm{H}_{2} \mathrm{O}, \mathrm{AgNO}_{3}, \mathrm{~Pb}\left(\mathrm{NO}_{3}\right)_{2}, \mathrm{KNO}_{3}$, $\mathrm{Co}\left(\mathrm{NO}_{3}\right)_{2} \cdot 6 \mathrm{H}_{2} \mathrm{O}, \mathrm{Cd}\left(\mathrm{NO}_{3}\right)_{2} \cdot 4 \mathrm{H}_{2} \mathrm{O}$, and $\mathrm{Hg}\left(\mathrm{NO}_{3}\right)_{2} \cdot \mathrm{H}_{2} \mathrm{O}$ were all purchased from Sinopharm Chemical Reagent Co., Ltd. (Shanghai, China). Quinine sulfate was purchased from Aladdin Chemistry Co., Ltd. All the chemicals were of analytical grade and were used as received without any further purification.
High-purity deionized water with a resistivity of greater than 18 $\mathrm{M} \Omega \mathrm{cm}^{-1}$ was obtained from the Millipore system and was used throughout the experiments.

\section{Preparation of CDs}

The CDs were prepared via a one-step hydrothermal method, $1.5 \mathrm{~g}$ of citric acid (CA) and $0.45 \mathrm{~g}$ of polyvinyl pyrrolidone (PVP $\mathrm{K}-30$ ) were dissolved in $15 \mathrm{ml}$ of deionized water to form a transparent viscous solution. Then, the mixture solution was transferred to a Teflon-lined autoclave $(20 \mathrm{ml})$ and heated at $180^{\circ} \mathrm{C}$ for $6 \mathrm{~h}$. After cooling to room temperature gradually, the obtained reactor was precipitated with the addition of excess acetone. The suspension was then centrifuged twice at $8000 \mathrm{rpm}$ for $20 \mathrm{~min}$; a yellow solution was obtained after removing the insoluble substances. After being kept stationary for half a day to evaporate the acetone, the solution was put in a freezing chamber of the fridge and was then freeze-dried into a powder in the freeze drier. Finally, the as-prepared pale yellow CDs powder was obtained, and an aqueous solution of $3 \mathrm{mg}$ $\mathrm{ml}^{-1}$ was also prepared for subsequent uses.

\section{Measurements}

The fluorescence spectra (PL) were obtained using an RF5301PC fluorescence spectrophotometer (Shimadzu, Japan). Transmission electron microscopy (TEM) images were obtained on a Hitachi-7650 transmission electron microscope (Hitachi, Japan). X-ray photoelectron spectroscopic (XPS) analyses were performed on a Wscalab X-ray photoelectron spectrometer (ThermoFisher, UK). Fourier transform infrared spectra (FTIR) were recorded on a Nicolet iS10 FTIR spectrometer (Thermo Scientific, USA). Ultraviolet-visible (UV-vis) absorption spectra were performed on a UV-3600 UV-vis spectrophotometer (Shimadzu, Japan).

\section{Fluorescent quantum yield (QY) measurement}

The QY of the as-prepared CDs was measured by the slope method as done in previous research..$^{33} \mathrm{~A}$ solution of quinine sulfate in $0.1 \mathrm{~mol} \mathrm{~L}^{-1} \mathrm{H}_{2} \mathrm{SO}_{4}$ (QY of $54 \%$ at $360 \mathrm{~nm}, \eta=1.33$ ) was used as the standard. The value of the quantum yield was calculated according to the following equation:

$$
Q_{x}=Q_{\mathrm{st}}\left(K_{x} / K_{\mathrm{st}}\right)\left(\eta_{x} / \eta_{\mathrm{st}}\right)^{2}
$$

where $Q$ is QY, $K$ is the slope, $\eta$ is the refractive index with 1.33 as the default for both quinine sulfate and CDs. The subscript 'st' refers to the quinine sulfate, while the subscript ' $x$ ' refers to the samples.

\section{Metal ion detection}

The metal ion detection procedures were performed in an aqueous solution of the as-prepared CDs at RT. $50 \mathrm{ml}$ of CDs solution was added to a colorimetric tube, a blank sample was set up, and the other samples were added into salt solutions (4 $\mathrm{mM}$ ) separately containing different metal ions of $\mathrm{Cu}^{2+}, \mathrm{Mn}^{2+}$, $\mathrm{Ca}^{2+}, \mathrm{Zn}^{2+}, \mathrm{Fe}^{2+}, \mathrm{Fe}^{3+}, \mathrm{Na}^{+}, \mathrm{Mg}^{2+}, \mathrm{Ni}^{2+}, \mathrm{Ag}^{+}, \mathrm{Pb}^{2+}, \mathrm{K}^{+}, \mathrm{Co}^{2+}, \mathrm{Cd}^{2+}$, $\mathrm{Hg}^{2+}$ with full stirring. After stirring for 5 minutes, the 
fluorescence intensity was recorded at an excitation wavelength of $360 \mathrm{~nm}$. The slit for the excitation was $1.5 \mathrm{~nm}$, and the emission was $1.5 \mathrm{~nm}$. All the experimental procedures were carried out under the same conditions. The detection of $\mathrm{Fe}^{3+}$ was examined using the same procedure by adding different concentrations of $\mathrm{Fe}^{3+}$ into aqueous solutions of CDs.

\section{Fluorescent ink}

A filter paper upon which the CDs adhered well (the paper showed no background under UV fluorescence) was chosen as the printing paper. Colorless CDs aqueous solutions were dipped with a brush pen to write different graphic patterns on the filter paper.

\section{Results and discussion}

\section{Composition and structure of CDs}

In this work, the CDs were synthesized by a hydrothermal reaction with the raw materials of citric and polyvinyl pyrrolidone at $180{ }^{\circ} \mathrm{C}$ for 6 hours. Under the hydrothermal environment of high temperature and high pressure, the water evaporation spurs the intermolecular dehydration of citric acid and polyvinyl pyrrolidone molecules to form "polymer-like" intermediates. ${ }^{34}$ The intermediates were further carbonized and capped by polyvinyl pyrrolidone molecules simultaneously to form the scattered carbon dots.

The morphology and microstructure of the as-prepared CDs were identified by TEM. As shown in Fig. 1a and b, the obtained CDs are spherical in structure, with a uniform dispersion without apparent aggregation. In the high-resolution TEM spectrum (Fig. 1c), no obvious lattice fringes were observed, indicating that the carbon quantum dots have poor crystallinity. Fig. 1d shows the size distribution histograms of the CDs, the distribution of the diameter size of the CDs within the range of $3.1-3.9 \mathrm{~nm}$, and the average particle diameter size is $3.4 \mathrm{~nm}$.

The surface functional groups and chemical composition of the CDs were identified by FTIR spectra and X-ray photoelectron spectroscopy (XPS). The FTIR spectra of the as-prepared CDs and its precursors are shown in Fig. 2, the wide band within the range of $3300 \mathrm{~cm}^{-1}$ to $3380 \mathrm{~cm}^{-1}$ can be attributed to $\mathrm{O}-\mathrm{H}$ and $\mathrm{N}-\mathrm{H}$ stretching vibrations. ${ }^{35}$ The characteristic peaks at $2970-$ $2890 \mathrm{~cm}^{-1}$ and $1725-1680 \mathrm{~cm}^{-1}$ are the stretching vibrations of $\mathrm{C}-\mathrm{H}$ and $\mathrm{C}=\mathrm{C}$, respectively. The characteristic peaks at $1450-$ $1380 \mathrm{~cm}^{-1}$ and $1070 \mathrm{~cm}^{-1}$ were assigned to the bending vibrations of $\mathrm{C}-\mathrm{N}, \mathrm{N}-\mathrm{H}, \mathrm{COO}$ and the $\mathrm{C}-\mathrm{O}, \mathrm{C}-\mathrm{O}-\mathrm{C}$ groups,
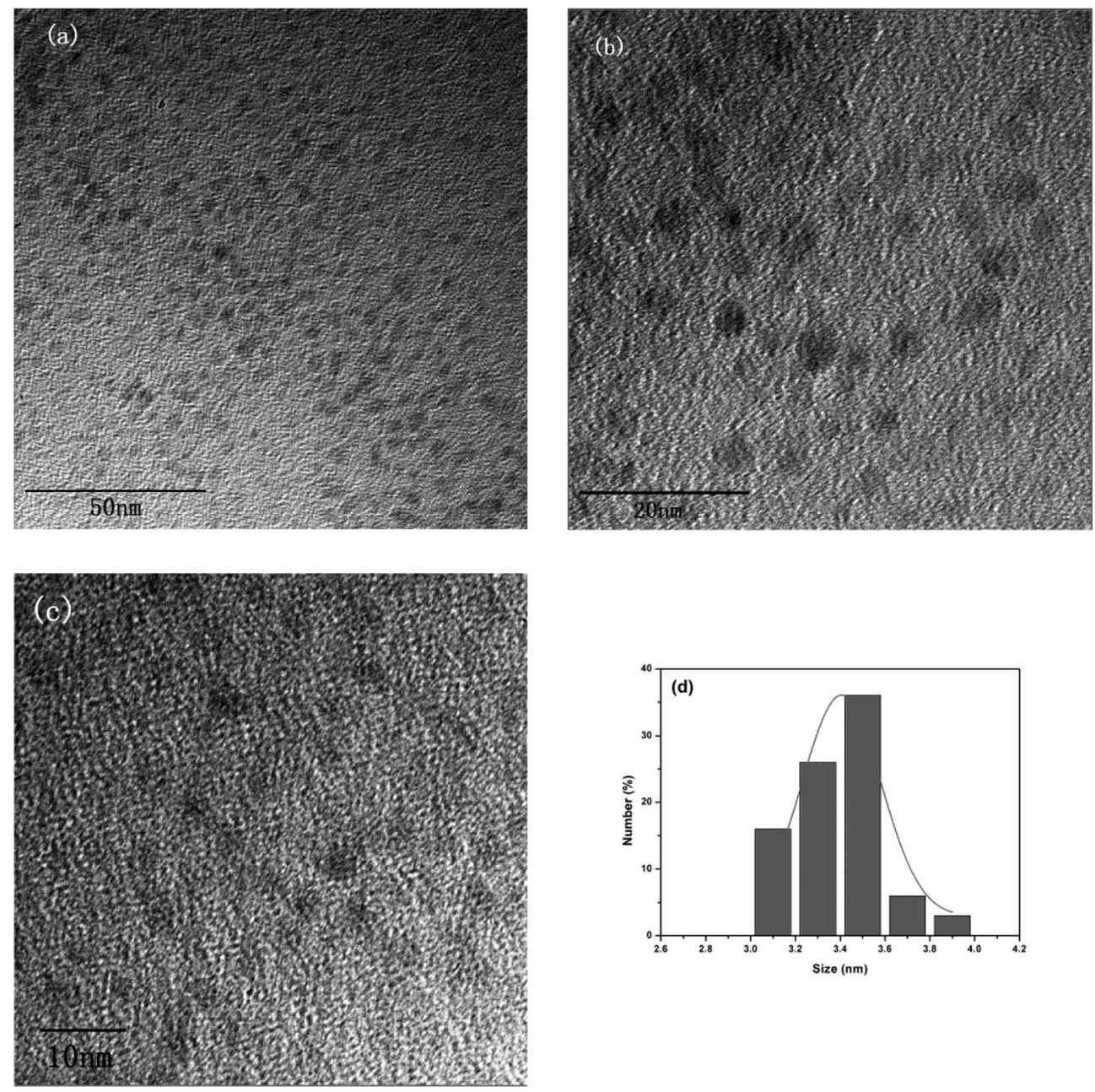

Fig. 1 (a) and (b) TEM images of the CDs; (c) high-resolution TEM images of the CDs; (d) and size distribution histograms of the CDs. 


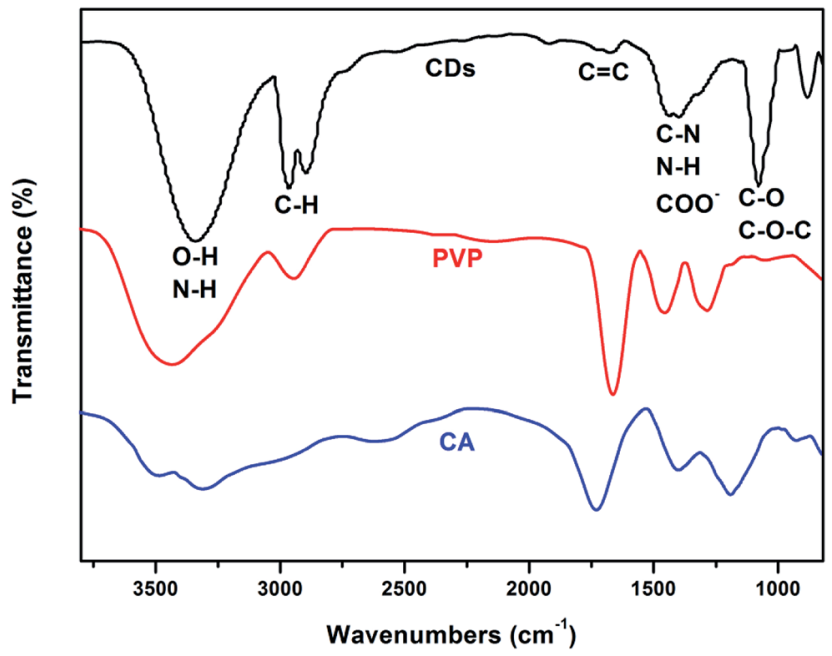

Fig. 2 FTIR spectra of the as-prepared CDs, PVP and citric acid, respectively.

respectively. What's more, compared with the FTIR spectra of the as-prepared CDs and the raw materials of PVP and citric acid (Fig. 4), we can find that the FTIR spectrum of the as-prepared CDs is almost the same as that of PVP, which can be ascribed to how the surface of CDs may be wrapped with abundant PVP long chains. However, the characteristic absorption band at 1639 and $1274 \mathrm{~cm}^{-1}$ corresponding to the acid amides and the
$\mathrm{CH}_{2}$ stretching vibration of PVP are absent in the FTIR spectrum of CDs, which suggests that the PVP units in CDs were changed. Similarly, the characteristic absorption peaks of citric acid also changed in the CDs, which further confirmed that a chemical reaction took place between the reactants, not just a simple physical interaction. The synthesis process of the CDs is schematically illustrated in Scheme 1. There are abundant active groups like carboxyl and hydroxyl on the surface of the citric acid, which are beneficial for improved water-solubility and also provide more reactivity sites. The polyvinyl pyrrolidone introduces different surface functional groups that would create new surface electronic energy levels and various characteristics. Meanwhile, the polyvinyl pyrrolidone provides abundant nitrogen-containing functional groups, which increases the fluorescence quantum yield (QY) and modifies the photoelectric properties. ${ }^{36}$

The surface composition of the synthesized CDs was examined with X-ray photoelectron spectroscopy (XPS) spectra, which are shown in Fig. 3. The XPS spectrum includes three peaks at 533.1, 400.1 and $285.1 \mathrm{eV},{ }^{37,38}$ which correspond to C1s, N1s and O1s, respectively (Fig. 3a). The sample contains 69.88 at\% of carbon, 7.80 at $\%$ of nitrogen and 22.32 at\% of oxygen. The corresponding binding energies were given in Fig. 3a, which indicated the existence of large amounts of carbon and oxygen elements and small amounts of nitrogen elements on the surface of the CDs. The appearance of the N1s peak indicates
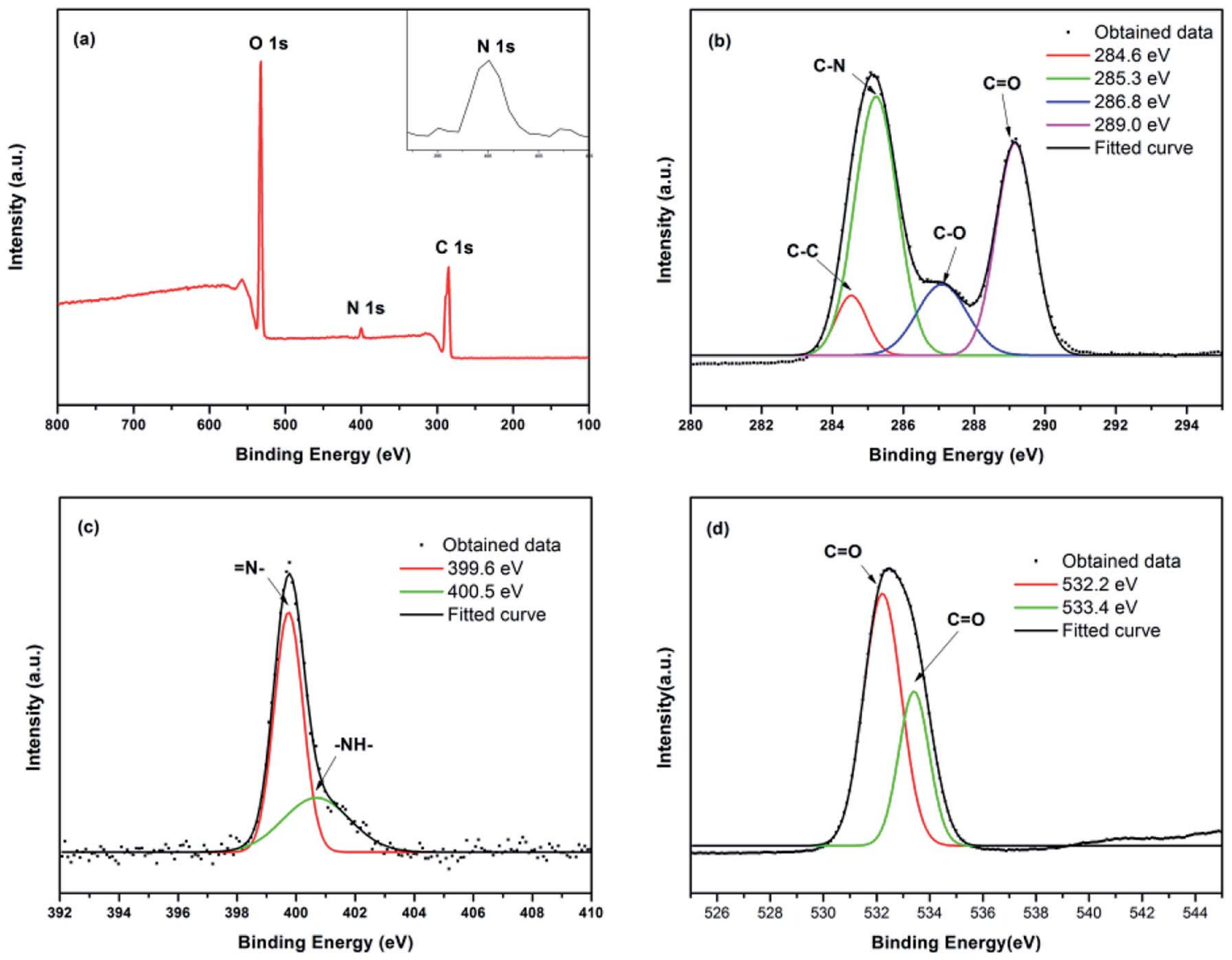

Fig. 3 (a) XPS survey spectrum, magnification and fitting curves of (b) the C1s peak, (c) the N1s peak, and (d) the O1s peak for the prepared CDs. 

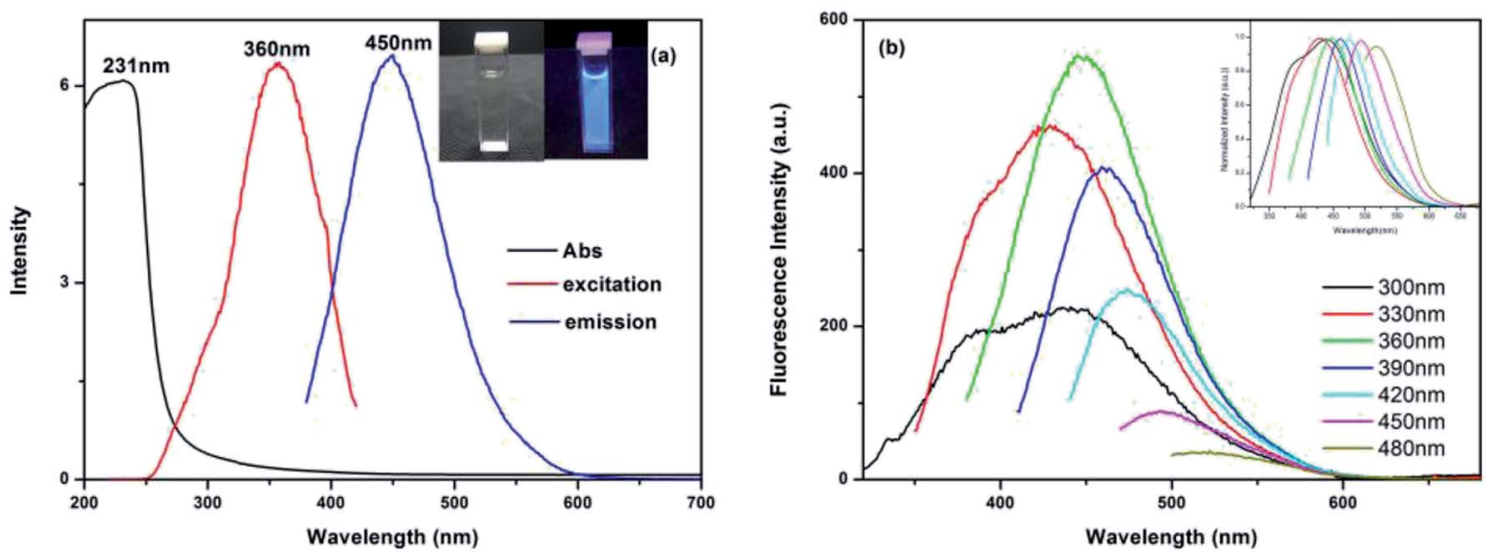

Fig. 4 (a) Absorbance, excitation and emission spectra of the CDs aqueous solution (the inset displays the fluorescent photo under a $365 \mathrm{~nm}$ UV lamp); (b) emission spectra of the CDs aqueous solution at different excitation wavelengths from 200 to $480 \mathrm{~nm}$ (the inset displays the corresponding normalized spectra).

that the $\mathrm{N}$ elements had successfully entered the carbon skeleton. Fig. $3 \mathrm{~b}$ shows the C1s spectrum of CDs; the four peaks at $284.6 \mathrm{eV}, 285.3 \mathrm{eV}, 286.8 \mathrm{eV}$ and $289.0 \mathrm{eV}$ should be attributed to $\mathrm{C}-\mathrm{C}, \mathrm{C}-\mathrm{N}, \mathrm{C}-\mathrm{O}$ and $\mathrm{C}=\mathrm{O} .{ }^{39}$ The high-resolution $\mathrm{N} 1 \mathrm{~s}$ spectra (Fig. 3c) of the obtained CDs present two kinds of main peaks associated with nitrogen atoms located at $399.6 \mathrm{eV}$ and $400.5 \mathrm{eV}$, which correspond to the pyridinic $\mathrm{N}$ of $=\mathrm{N}-$ and the pyrrolic $\mathrm{N}$ of $-\mathrm{NH}-$, respectively. As previously reported, the pyrrolic $\mathrm{N}$ exposes the edge of the plane and the defect position, and the pyridinic $\mathrm{N}$ replace $\mathrm{C}$ atoms at the edge or plane of the defective parts. ${ }^{40,41}$ The defects and planes caused by $\mathrm{N}$ doping can significantly improve the surface chemical activity and electrical properties of CDs and then improve the QY of the CDs. The O1s spectrum of the CDs is shown in Fig. 3d. The two main peaks at $532.2 \mathrm{eV}$ and $533.4 \mathrm{eV}$ could be assigned to $\mathrm{C}-\mathrm{O}$ and $\mathrm{C}=\mathrm{O}$. The XPS results agreed with FTIR analysis. In other words, there may be hydrophilic groups such as $-\mathrm{OH},-\mathrm{NH}_{2}$ and $-\mathrm{COOH}$ on the surface of the obtained CDs.

\section{Optical properties of CDs}

We used the slope method to measure the QY of the as-prepared CDs. The relative fluorescence QY of the CDs was calculated to be $30.21 \%$ using quinine sulfate in $0.1 \mathrm{~mol} \mathrm{~L}^{-1} \mathrm{H}_{2} \mathrm{SO}_{4}$ (QY of $54 \%$ at $360 \mathrm{~nm}, \eta=1.33$ ) as the reference. The UV-vis absorption spectrum is shown in Fig. 4a. The absorption band ranged from 200 to $280 \mathrm{~nm}$ with a peak at $231 \mathrm{~nm}$, which can be assigned to the $\pi-\pi^{*}$ transition of $\mathrm{C}=\mathrm{C} \mathrm{sp}^{2}$. The obtained CDs have the strongest blue emission band at $450 \mathrm{~nm}$ with an excitation at $360 \mathrm{~nm}$ (the inset picture of Fig. 4a). The PL emission wavelength of the CDs aqueous solution has a typical excitation-dependent property upon changing the excited wavelength as shown in Fig. 4b, which is common in fluorescent carbon materials. ${ }^{42}$ As the excitation wavelength increases from 300 to $480 \mathrm{~nm}$, the position of the emission peak shifts to long wavelengths from 425 to $520 \mathrm{~nm}$. Meanwhile, the PL intensity of the CDs reaches a maximum at $450 \mathrm{~nm}$ when excited at $360 \mathrm{~nm}$. The red shift of the emission band may be attributed to the surface state of the CDs that affect the band gap $^{43}$ or the effects of different emissive sites on the different sized nanoparticles within the sample. ${ }^{44}$ Nevertheless, the PL intensity reduces as the half-peak width increases, which may be associated with the quantum effect and surface energy trap. ${ }^{45}$
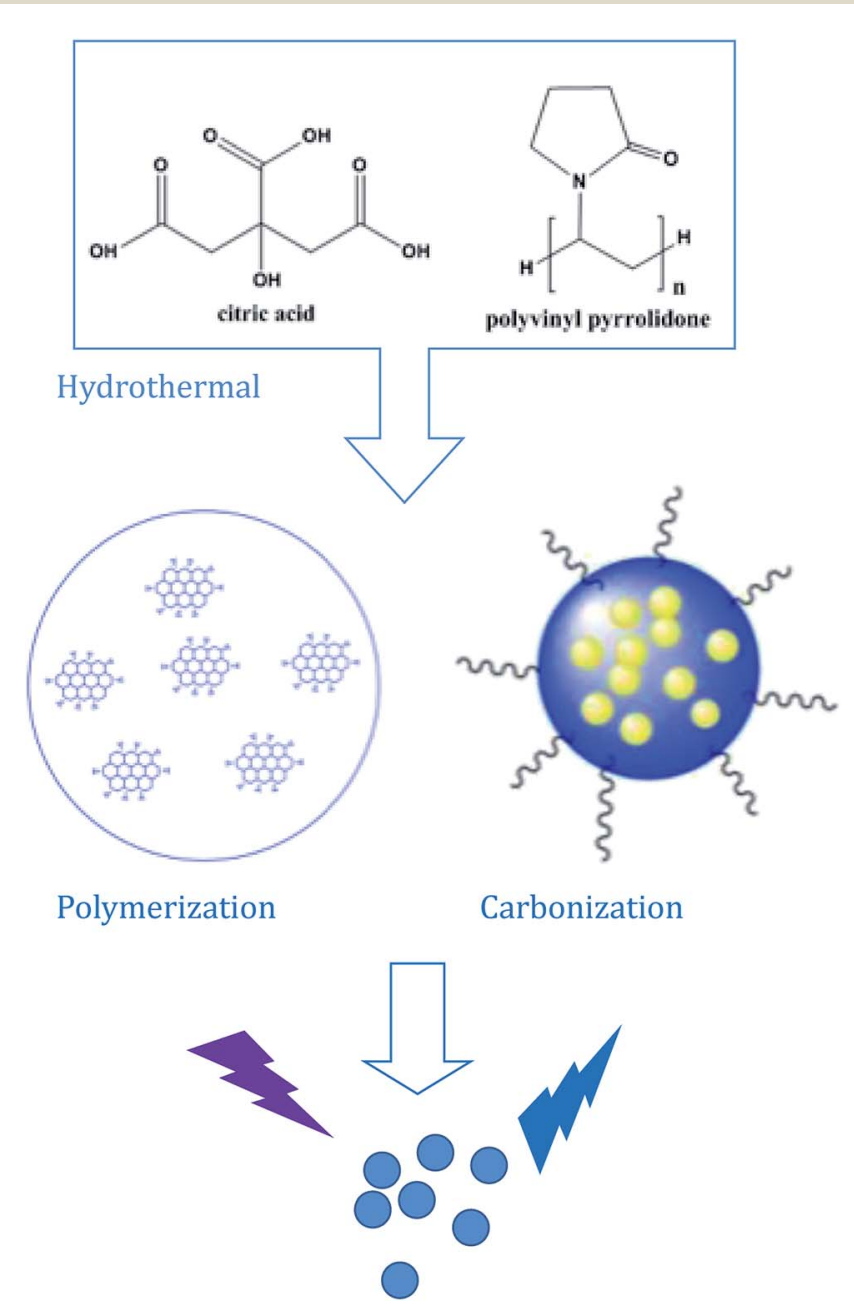

Scheme 1 A synthetic route using citric acid and polyvinyl pyrrolidone (PVP K-30): from ionization to condensation, polymerization, and carbonization. 
Furthermore, the quantum yield of the obtained CDs is as high as $32.1 \%$ (excited at $360 \mathrm{~nm}$ and using quinine sulfate as a reference), which is higher than in many previous reports. ${ }^{46,47}$

As we all know, the fluorescence stability of CDs is a key factor in its application in various fields. Therefore, the PL stability of the CDs was also studied (Fig. 5). As shown in Fig. 5a, after irradiation under a UV lamp (365 nm) for $1800 \mathrm{~s}$, the PL intensity of the CDs aqueous solution did not obviously change, which indicates that the CDs has good PL stability and have a potential application in biological detection, which may be needed for long-term radiation. What's more, in order to investigate the effect of $\mathrm{pH}$ on the PL intensity of CDs, we characterized the PL intensity of CDs aqueous solution with a $\mathrm{pH}$ value of $1,2,3,4,5,7,9,11$ and 12 , respectively. As shown in Fig. 5b, with the increase of $\mathrm{pH}$ value, the PL intensity first increases and then decreases. That is, the PL intensity of the CDs dispersing in the acid medium is stronger than that in the alkaline medium, and the PL intensity reaches a maximum near the $\mathrm{pH}$ value of 4 . By adjusting the $\mathrm{pH}$ value of the CDs solution, we found that the PL intensity changes with the $\mathrm{pH}$ value, which demonstrates that the as-prepared CDs has $\mathrm{pH}$-dependence. The result is common in the previous reports, which is relevant to the protonation/non-protonation of the surface groups $\left(-\mathrm{NH}_{2},-\mathrm{COOH},-\mathrm{C}=\mathrm{O}-\right) .{ }^{48}$

\section{Applications of CDs in ions detection}

The influence of the quenching of different ions on the fluorescence of CDs indicates the as-prepared CDs as a promising fluorescent probe for metal ion detection. The emission spectra of the CDs under $360 \mathrm{~nm}$ excitation with different metal ions were recorded after incubation for $10 \mathrm{~min}$, as shown in Fig. 6a. The detection solution included $\mathrm{Cu}^{2+}, \mathrm{Mn}^{2+}, \mathrm{Ca}^{2+}, \mathrm{Zn}^{2+}, \mathrm{Fe}^{2+}$, $\mathrm{Fe}^{3+}, \mathrm{Na}^{+}, \mathrm{Mg}^{2+}, \mathrm{Ni}^{2+}, \mathrm{Ag}^{+}, \mathrm{Pb}^{2+}, \mathrm{K}^{+}, \mathrm{Co}^{2+}, \mathrm{Cd}^{2+}$, and $\mathrm{Hg}^{2+}$ ions; the emission spectra of all the metal ions did not significant changed except for the $\mathrm{Fe}^{3+}$ ions. Fig. $6 \mathrm{~b}$ shows the different PL intensity ratios $\left(F / F_{0}\right)$ of the CDs solutions in the presence and absence of various metal ions. From this figure, we can observe that the PL intensity ratio $\left(F / F_{0}\right)$ of $\mathrm{Fe}^{3+}$ ions is the lowest and that other metal ions show slight changes of PL intensity, which
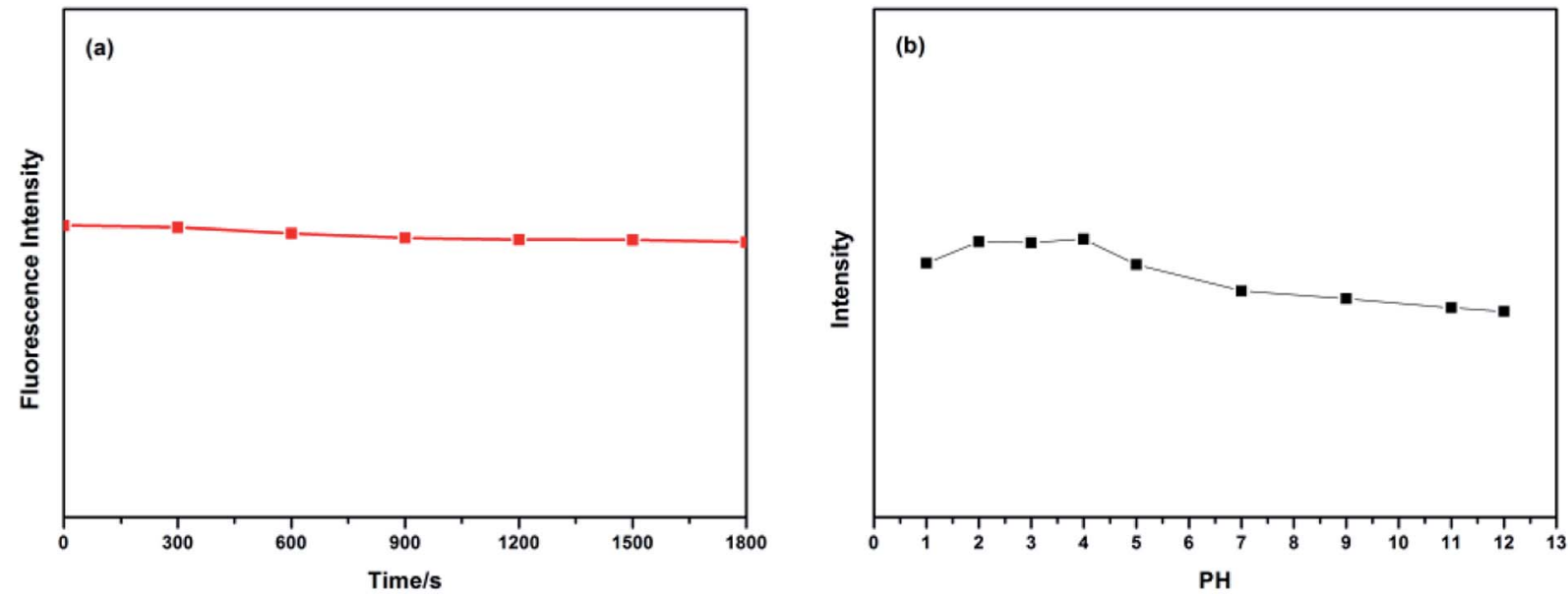

Fig. 5 (a) Irradiation time on the PL intensity of CDs and (b) the value on the PL intensity of CDs ( $\lambda_{\text {ex }}=360 \mathrm{~nm}$ ).
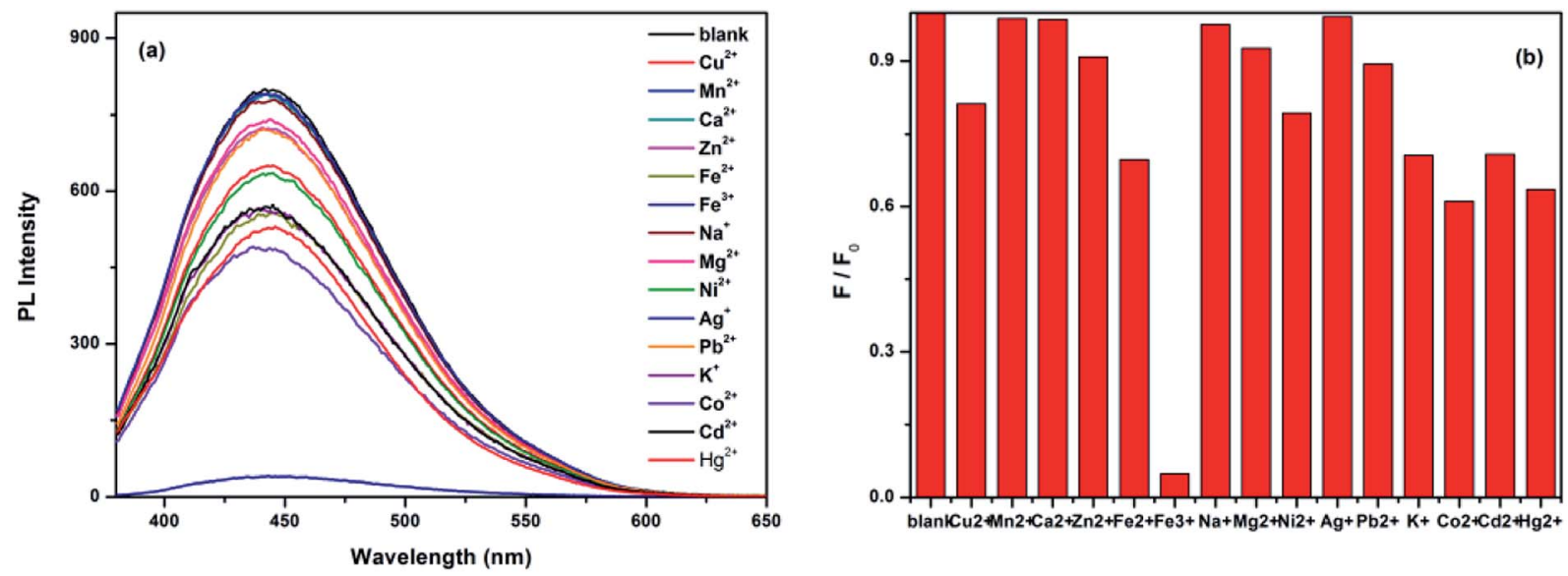

Fig. 6 (a) Emissions spectra of CDs with different ions; (b) the different $\mathrm{PL}$ intensity ratios $\left(F / F_{0}\right)$ of the $C D$ s solutions in the presence and absence of various metal ions ( $F_{0}$ is the blank value, $F$ is the fluorescence intensity of CDs with different ion, excitation $=360 \mathrm{~nm}$, emission $=450 \mathrm{~nm}$, $\left[\mathrm{Mn}^{+}\right]=4 \mathrm{mmol} \mathrm{L}{ }^{-1}$. 
indicated that $\mathrm{Fe}^{3+}$ has the largest quenching effect on the fluorescence of CDs and that the quenching effect is may be due to the strong affinity of $\mathrm{Fe}^{3+}$ ions to CDs. The results of FTIR and XPS confirmed that part of the $-\mathrm{COOH},-\mathrm{OH}$ and $-\mathrm{NH}_{2}$ groups exists on the surface of the as-prepared CDs, which can react with $\mathrm{Fe}^{3+}$ ions to form a complex and effectively prevents the hydrolyzing of $\mathrm{Fe}^{3+}$ in solution, finally leading to the fluorescence quenching of CDs. Moreover, variations on the surface of CDs may cause the aggregation of CDs, leading to fluorescence quenching. This phenomenon is agreed with the reported work, which had revealed that after the addition of $\mathrm{Fe}^{3+}$ to the CDs solution, the surface of CDs might be changed, which would result in poor luminescence efficiency. ${ }^{\mathbf{4 9 , 5 0}}$

At room temperature, the emission spectra of the CDs in the presence of different concentrations of $\mathrm{Fe}^{3+}$ ions were recorded after incubation for $10 \mathrm{~min}$. As shown in Fig. 7a, after excitation at $360 \mathrm{~nm}$, CDs solutions with different concentrations of $\mathrm{Fe}^{3+}$ ions show a broad band with a peak at $450 \mathrm{~nm}$, ranging from 380 to $600 \mathrm{~nm}$ with an increasing concentration of $\mathrm{Fe}^{3+}$ ions. The fluorescence intensity at $450 \mathrm{~nm}$ decreases gradually without any red or blue shift in position, which indicates that the quenching effect is related to the concentration of $\mathrm{Fe}^{3+}$; CDs, therefore, can be used as the probe of $\mathrm{Fe}^{3+}$ ions. The $\mathrm{Fe}^{3+}$ quenching effect can be described with the Stern-Volmer equation:

$$
F_{0} / F-1=K_{\mathrm{sv}} C
$$

where $K_{\mathrm{sv}}$ is the Stern-Volmer quenching constant and the bimolecular quenching constant, $C$ is the concentration of $\mathrm{Fe}^{3+}$, and $F_{0}$ and $F$ are the fluorescence intensity of CDs without $\mathrm{Fe}^{3+}$ and with different concentration of $\mathrm{Fe}^{3+}$, respectively. Fig. $7 \mathrm{~b}$ shows the standard curve of $F_{0} / F$ to the concentration of $\mathrm{Fe}^{3+}$; the range of linearity is $0-300 \mu \mathrm{mol}$, the corresponding regression coefficient $R^{2}=0.991$, and the detection limit (LOD) for $\mathrm{Fe}^{3+}$ is $45.5 \mathrm{nmol} \mathrm{L}{ }^{-1}$ based on $3 \sigma / k$ ( $\sigma$ represents the standard deviation for the corrected blank signals with 10 parallel measurements, and $k$ is the slope of the calibration curve). Moreover, on/off fluorescence was achieved by oxidizing $\mathrm{Fe}^{2+}$ to $\mathrm{Fe}^{3+}$ in such an imaging system (Fig. 7c and d).

\section{Solid-state fluorescence of CDs}

Furthermore, the self-quenching-resistant property of the asprepared CDs was founded after the freeze-drying process. Excited by UV light (365 nm), the CDs powder exhibits strong blue solid-state fluorescence (SSF), as shown in Fig. 8. The SSF is attributed to the space steric hindrance caused by the PVP chains on the surface of the CDs particles, which was confirmed by FTIR spectroscopy. The long chains prevent the graphitized carbon cores from directly contacting each other, such that keeping a certain distance among the CDs particles allows for the avoidance of the self-quenching effect generated by $\pi-\pi$ stacking (one kind of efficient energy transfer mode), which shows as the solid-state fluorescence. ${ }^{21}$

Furthermore, the emission spectra of the CDs powder are shown in Fig. 9. The PL emission wavelength of the CDs powder has an excitation-dependent property when changing the excitation wavelength from 330 to $600 \mathrm{~nm}$, similar to the CDs aqueous solution. As the excitation wavelength increases, the

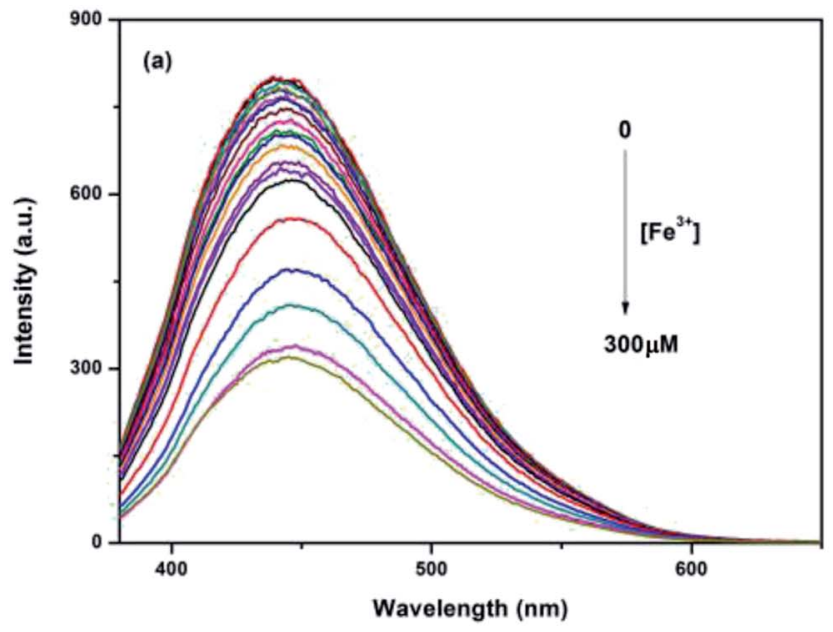

(c)

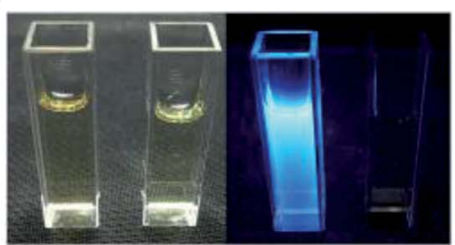

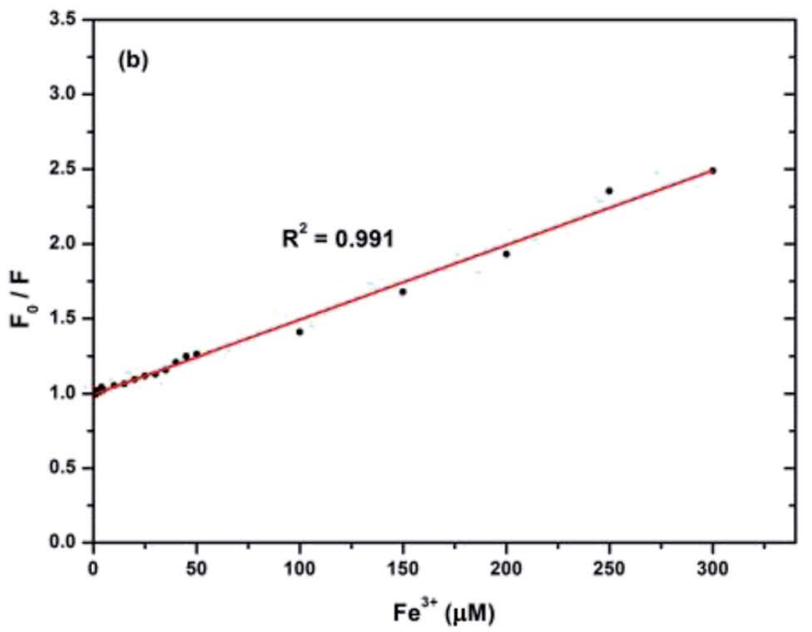

(d)

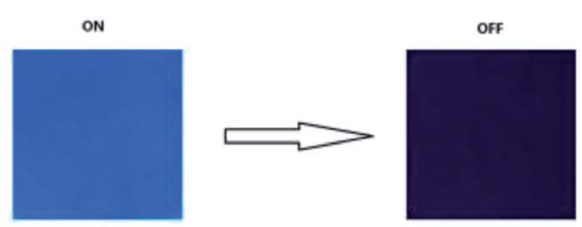

Fig. 7 (a) Quenching curves of CDs upon exposure to different concentration of $\mathrm{Fe}^{3+}$; (b) the linear region of the $\mathrm{CDs}$ - $\mathrm{Fe}^{3+}$ sensing response ( $\left[\mathrm{Fe}^{3+}\right]$ : $0.0,1.0,2.0,3.0,4.0,5.0,10,15,20,25,30,35,40,45,50,100,150,200,250$ and $300 \mu \mathrm{mol} \mathrm{L}^{-1}$, respectively); (c) images of the CDs before (left) and after (right) treatment with $\mathrm{Fe}^{3+}$ under sunlight and UV light, respectively; (d) fluorescence images of aqueous $\mathrm{CDs}$ after adding Fe ${ }^{2+}$ and $\mathrm{Fe}^{3+}$ ions; the on/off fluorescence was achieved by oxidation. 


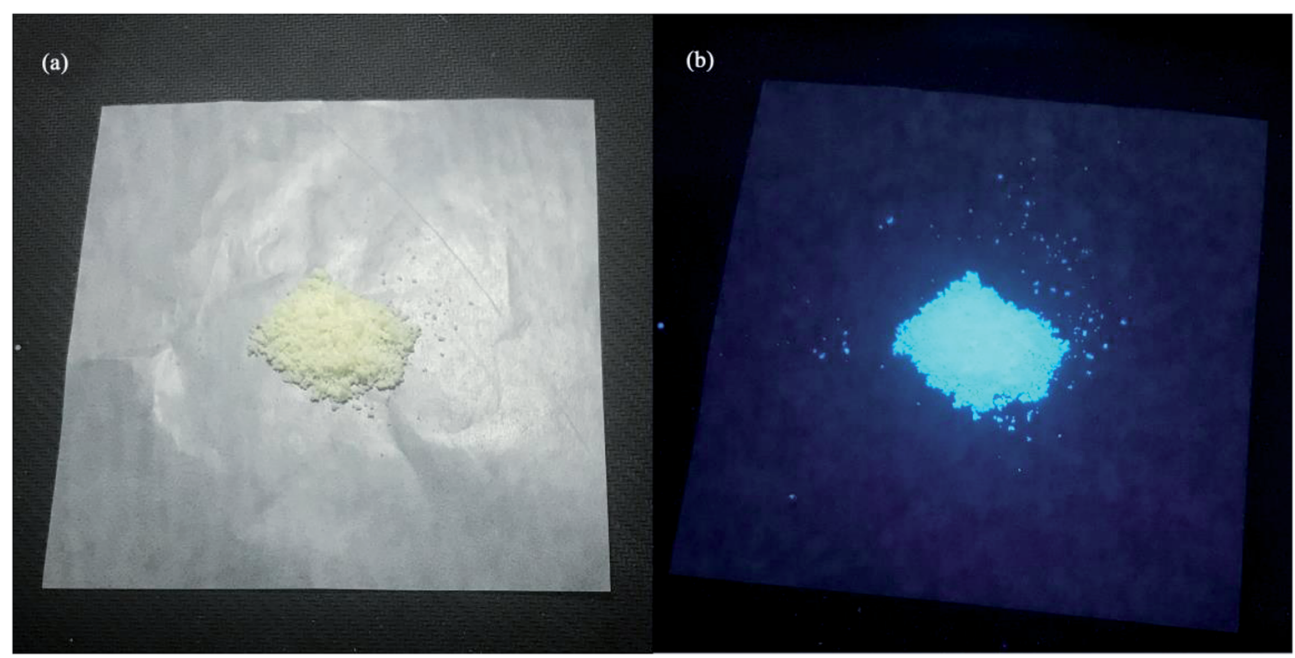

Fig. 8 Photographs of CDs were taken under (a) daylight and (b) $365 \mathrm{~nm}$ UV light.

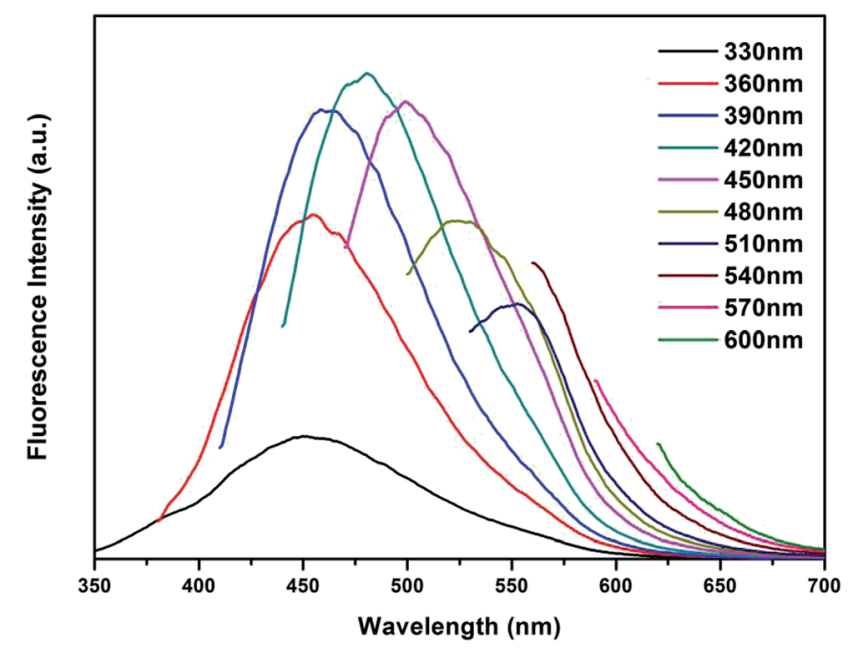

Fig. 9 SSF spectra of CDs powder at different excitation wavelengths from 330 to $600 \mathrm{~nm}$. position of the emission peak of the CDs powder shifts to longer wavelengths from 451 to $600 \mathrm{~nm}$, while the PL intensity of the CDs reaches the maximum at $482 \mathrm{~nm}$ when excited at $420 \mathrm{~nm}$. The self-quenching-resistance property of the CDs powder may provide an opportunity for the achievement of efficient SSF nanomaterials without being supported by solid matrices.

\section{Application of CDs in fluorescent ink}

For its excellent fluorescence, photobleaching resistance, good PL stability and good dispersibility, aqueous CDs can be used as fluorescent ink. The fluorescent characters written on paper by using CDs ink are not visible, except when excited under a UV lamp. As shown in Fig. 10a, "Shanghai University" CDs blue fluorescent characters can be observed under a UV light. A month later, the characters still emit a clear and intense blue light under $365 \mathrm{~nm}$ UV excitation. When the concentration of the CDs in aqueous solution changed, the CDs ink showed different shades of blue under the UV lamp (Fig. 10b). High- (a)

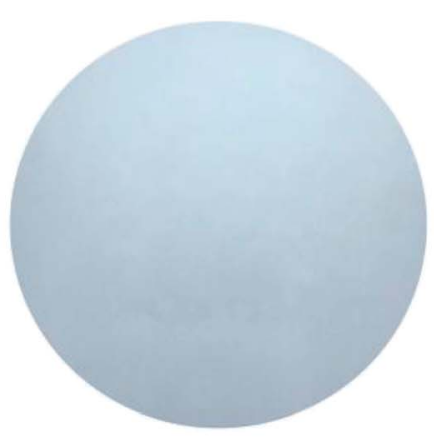

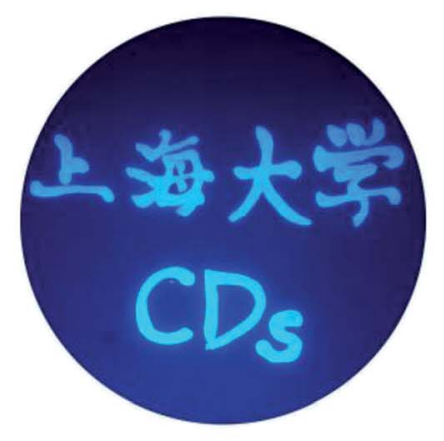

(b)

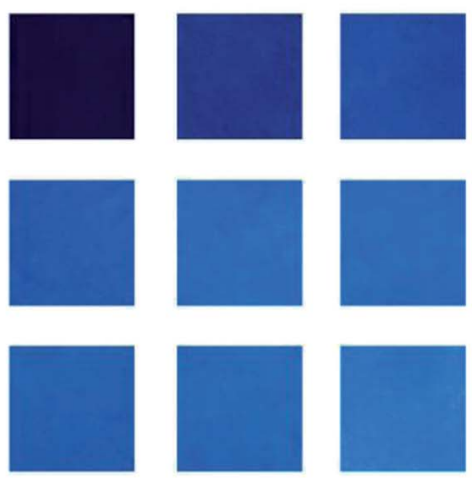

Fig. 10 (a) Different graphic patterns on paper (illuminated by the $365 \mathrm{~nm}$ UV lamp) and (b) ink in multiple colors, tuned by the CDs concentration in aqueous solution (illuminated by the $365 \mathrm{~nm}$ UV lamp). 
quality fluorescent graphics and text characters can be obtained by using CDs fluorescent ink, which can replace traditional ink for anti-counterfeiting, information encryption, information storage and so on. In the near future, the CDs fluorescent ink may have great application in flexible displays.

\section{Conclusion}

In summary, the blue CDs were successfully synthesized by a simple one-pot hydrothermal treatment by using citric acid (carbon source) and polyvinyl pyrrolidone (PVP K-30) (nitrogen source) as the raw materials. The QY of the as-prepared CDs is $30.21 \%$, and it has remarkable water-dispensability and excellent fluorescence stability. The obtained CDs have the strongest blue emission band at $450 \mathrm{~nm}$ with an excitation at $360 \mathrm{~nm}$. The emission spectra of the obtained CDs exhibited an excitationdependent fluorescence behavior with a center of broad range shifts from 425 to $520 \mathrm{~nm}$. The CDs could be used to detect the $\mathrm{Fe}^{3+}$ ions within a linear range of $0-300 \mu \mathrm{M}$, with a detection limit of $45.5 \mathrm{nmol} \mathrm{L}^{-1}$, which suggested that the new CDs has great potential toward the detection of $\mathrm{Fe}^{3+}$ ions. What's more, the excellent self-quenching-resistant property of the asprepared CDs was showed in the solid state, with bright blue fluorescence being observed under a $365 \mathrm{~nm}$ UV lamp, providing more possibilities for the solid application of CDs. Besides, the CDs could be applied as a fluorescent ink that could potentially be a substitute for commercial dyes. All of the results suggest the as-prepared CDs as a new class of luminescent carbon nanomaterials with great potential in environmental and commercial applications in the near future.

\section{Conflicts of interest}

There are no conflicts to declare.

\section{Acknowledgements}

The author is grateful to the National Natural Science Foundation (Grant 21301115), and the project is supported by the Key Laboratory for Advanced Displays and System Application, Ministry of Education, Shanghai University (Grant P201602).

\section{References}

$1 \mathrm{X} . \mathrm{Xu}, \mathrm{R} . \mathrm{Ray}, \mathrm{Y} . \mathrm{Gu}$, et al., Electrophoretic Analysis and Purification of Fluorescent Single-Walled Carbon Nanotube Fragments, J. Am. Chem. Soc., 2004, 126(40), 12736-12737.

2 C. Zhao, Y. Jiao, F. Hu, et al., Green synthesis of carbon dots from pork and application as nanosensors for uric acid detection, Spectrochim. Acta, Part A, 2018, 190, 360-367.

3 P. G. Luo, S. Sahu, S.-T. Yang, et al., Carbon "quantum" dots for optical bioimaging, J. Mater. Chem. B, 2013, 1(16), 2116.

4 Y. P. Sun, B. Zhou, Y. Lin, et al., Quantum-Sized Carbon Dots for Bright and Colorful Photoluminescence, J. Am. Chem. Soc., 2006, 128(24), 7756-7757.
5 G. E. LeCroy, S. K. Sonkar, F. Yang, et al., Toward Structurally Defined Carbon Dots as Ultracompact Fluorescent Probes, ACS Nano, 2014, 8(5), 4522-4529.

6 Y. Song, S. Zhu and B. Yang, Bioimaging based on fluorescent carbon dots, RSC Adv., 2014, 4(52), 27184-27200.

7 H. Huang, Y. Xu, C. J. Tang, et al., Facile and green synthesis of photoluminescent carbon nanoparticles for cellular imaging, New J. Chem., 2014, 38(2), 784-789.

8 S. Zhu, Q. Meng, L. Wang, et al., Highly photoluminescent carbon dots for multicolor patterning, sensors, and bioimaging, Angew. Chem., Int. Ed. Engl., 2013, 52(14), 3953-3957.

$9 \mathrm{~W}$. Wei, C. Xu, L. Wu, et al., Non-enzymatic-browningreaction: a versatile route for production of nitrogen-doped carbon dots with tunable multicolor luminescent display, Sci. Rep., 2014, 4, 3564.

10 S. Qu, X. Wang, Q. Lu, et al., A biocompatible fluorescent ink based on water-soluble luminescent carbon nanodots, Angew. Chem., Int. Ed. Engl., 2012, 51(49), 12215-12218.

11 Y. Chen, M. Zheng, Y. Xiao, et al., A Self-Quenching-Resistant Carbon-Dot Powder with Tunable Solid-State Fluorescence and Construction of Dual-Fluorescence Morphologies for White Light-Emission, Adv. Mater., 2016, 28(2), 312-318.

12 L.-H. Mao, W.-Q. Tang, Z.-Y. Deng, et al., Facile Access to White Fluorescent Carbon Dots toward Light-Emitting Devices, Ind. Eng. Chem. Res., 2014, 53(15), 6417-6425.

13 X. Guo, C. F. Wang, Z. Y. Yu, et al., Facile access to versatile fluorescent carbon dots toward light-emitting diodes, Chem. Commun., 2012, 48(21), 2692-2694.

14 M. Shamsipur, A. Barati and S. Karami, Long-wavelength, multicolor, and white-light emitting carbon-based dots: achievements made, challenges remaining, and applications, Carbon, 2017, 124, 429-472.

15 H. Ali, S. K. Bhunia, C. Dalal, et al., Red Fluorescent Carbon Nanoparticle-Based Cell Imaging Probe, ACS Appl. Mater. Interfaces, 2016, 8(14), 9305-9313.

16 H. Huang, Y. C. Lu, A. J. Wang, et al., A facile, green, and solvent-free route to nitrogen-sulfur-codoped fluorescent carbon nanoparticles for cellular imaging, RSC Adv., 2014, 4(23), 11872-11875.

17 W. P. Wang, Y. C. Lu, H. Huang, et al., Facile synthesis of water-soluble and biocompatible fluorescent nitrogendoped carbon dots for cell imaging, Analyst, 2014, 139(7), 1692-1696.

18 W. J. Lu, X. J. Gong, Z. H. Yang, et al., High-quality watersoluble luminescent carbon dots for multicolor patterning, sensors, and bioimaging, RSC Adv., 2015, 5(22), 16972-16979.

19 Y. Q. Dong, R. X. Wang, H. Li, et al., Polyaminefunctionalized carbon quantum dots for chemical sensing, Carbon, 2012, 50(8), 2810-2815.

20 M. Xu, G. He, Z. Li, et al., A green heterogeneous synthesis of $\mathrm{N}$-doped carbon dots and their photoluminescence applications in solid and aqueous states, Nanoscale, 2014, 6(17), 10307-10315.

21 J. Zhu, X. Bai, Y. Zhai, et al., Carbon dots with efficient solidstate photoluminescence towards white light-emitting diodes, J. Mater. Chem. C, 2017, 5(44), 11416-11420. 
22 H. M. Zidan, E. M. Abdelrazek, A. M. Abdelghany, et al., Characterization and some physical studies of PVA/PVP filled with MWCNTs, J. Mater. Res. Technol., 2018, DOI: 10.1016/j.jmrt.2018.04.023.

23 X. Cui, Y. Wang, J. Liu, et al., Dual functional N- and S-codoped carbon dots as the sensor for temperature and $\mathrm{Fe}^{3+}$ ions, Sens. Actuators, B, 2017, 242, 1272-1280.

24 X. Qu, Q. Liu, X. Ji, et al., Enhancing the Stokes' shift of BODIPY dyes via through-bond energy transfer and its application for $\mathrm{Fe}^{3+}$-detection in live cell imaging, Chem. Commun., 2012, 48(38), 4600-4602.

25 P. Wu, Y. Li, X. P. Yan, et al., CdTe Quantum Dots (QDs) Based Kinetic Discrimination of $\mathrm{Fe}^{2+}$ and $\mathrm{Fe}^{3+}$, and CdTe QDs-Fenton Hybrid System for Sensitive Photoluminescent Detection of $\mathrm{Fe}^{2+}$, Anal. Chem., 2009, 81(15), 6252-6257.

26 L. Shang, L. Yang, F. Stockmar, et al., Microwave-assisted rapid synthesis of luminescent gold nanoclusters for sensing $\mathrm{Hg}^{2+}$ in living cells using fluorescence imaging, Nanoscale, 2012, 4(14), 4155-4160.

27 C. X. Yang, H. B. Ren, X. P. Yan, et al., Fluorescent MetalOrganic Framework MIL-53(Al) for Highly Selective and Sensitive Detection of $\mathrm{Fe}^{3+}$ in Aqueous Solution, Anal. Chem., 2013, 85(15), 7441-7446.

28 B. Shi, Y. Su, L. Zhang, et al., Nitrogen and Phosphorus CoDoped Carbon Nanodots as a Novel Fluorescent Probe for Highly Sensitive Detection of $\mathrm{Fe}(3+)$ in Human Serum and Living Cells, ACS Appl. Mater. Interfaces, 2016, 8(17), 10717-10725.

29 W. P. Wang, Y. H. Lu, H. Huang, et al., Solvent-free synthesis of sulfur- and nitrogen-co-doped fluorescent carbon nanoparticles from glutathione for highly selective and sensitive detection of mercury(II) ions, Sens. Actuators, B, 2014, 202, 741-747.

$30 \mathrm{H}$. Zhu, X. Wang, Y. Li, et al., Microwave synthesis of fluorescent carbon nanoparticles with electrochemiluminescence properties, Chem. Commun., 2009, 34, 5118-5120.

31 F. S. Niu, Y. L. Ying, X. Hua, et al., Electrochemically generated green-fluorescent $\mathrm{N}$-doped carbon quantum dots for facile monitoring alkaline phosphatase activity based on the $\mathrm{Fe}^{3+}$-mediating ON-OFF-ON-OFF fluorescence principle, Carbon, 2018, 127, 340-348.

32 Q. Xu, P. Pu, J. G. Zhao, et al., Preparation of highly photoluminescent sulfur-doped carbon dots for Fe(iii) detection, J. Mater. Chem. A, 2015, 3(2), 542-546.

33 M. Asha Jhonsi and S. Thulasi, A novel fluorescent carbon dots derived from tamarind, Chem. Phys. Lett., 2016, 661, 179-184.

34 W. Kwon, G. Lee, S. Do, et al., Size-controlled soft-template synthesis of carbon nanodots toward versatile photoactive materials, Small, 2014, 10(3), 506-513.

35 L. Wu, X. Cai, K. Nelson, et al., A Green Synthesis of Carbon Nanoparticle from Honey for Real-Time Photoacoustic Imaging, Nano Res., 2013, 6(5), 312-325.
36 Y. A. Wang, J. J. Li, H. Chen, et al., Stabilization of Inorganic Nanocrystals by Organic Dendrons, J. Am. Chem. Soc., 2002, 124(10), 2293-2298.

37 Y. Kuai, R. Fang, C. Zhang, et al., A convenient way of activating carbon quantum dots and the efficient isolation, Mater. Res. Bull., 2018, 104, 119-123.

38 A. B. Bourlinos, G. Trivizas, M. A. Karakassides, et al., Green and simple route toward boron doped carbon dots with significantly enhanced non-linear optical properties, Carbon, 2015, 83, 173-179.

39 M. A. Patel, F. Luo, M. R. Khoshi, et al., P-Doped Porous Carbon as Metal Free Catalysts for Selective Aerobic Oxidation with an Unexpected Mechanism, ACS Nano, 2016, 10(2), 2305-2315.

40 Q. Liang, W. Ma, Y. Shi, et al., Easy synthesis of highly fluorescent carbon quantum dots from gelatin and their luminescent properties and applications, Carbon, 2013, 60, 421-428.

41 J. Liu, D. Li, K. Zhang, et al., One-Step Hydrothermal Synthesis of Nitrogen-Doped Conjugated Carbonized Polymer Dots with 31\% Efficient Red Emission for In Vivo Imaging, Small, 2018, 14(15), 1703919.

42 J. Wang, F. Peng, Y. Lu, et al., Large-Scale Green Synthesis of Fluorescent Carbon Nanodots and Their Use in Optics Applications, Adv. Opt. Mater., 2015, 3(1), 103-111.

43 J. Shang, L. Ma, J. Li, et al., The origin of fluorescence from graphene oxide, Sci. Rep., 2012, 2, 792.

44 Y. Dong, N. Zhou, X. Lin, et al., Extraction of Electrochemiluminescent Oxidized Carbon Quantum Dots from Activated Carbon, Chem. Mater., 2010, 22(21), 58955899.

45 L. Cao, M. J. Meziani, S. Sahu, et al., Photoluminescence Properties of Graphene versus Other Carbon Nanomaterials, Acc. Chem. Res., 2013, 46(1), 171-180.

46 S. Sun, L. Zhang, K. Jiang, et al., Toward High-Efficient Red Emissive Carbon Dots: Facile Preparation, Unique Properties, and Applications as Multifunctional Theranostic Agents, Chem. Mater., 2016, 28(23), 8659-8668.

47 L. Shi, L. Li, X. Li, et al., Excitation-independent yellowfluorescent nitrogen-doped carbon nanodots for biological imaging and paper-based sensing, Sens. Actuators, B, 2017, 251, 234-241.

48 D. Sun, R. Ban, P.-H. Zhang, et al., Hair fiber as a precursor for synthesizing of sulfur- and nitrogen-co-doped carbon dots with tunable luminescence properties, Carbon, 2013, 64, 424-434.

49 G. Gao, Y.-W. Jiang, H.-R. Jia, et al., On-off-on fluorescent nanosensor for $\mathrm{Fe}^{3+}$ detection and cancer/normal cell differentiation via silicon-doped carbon quantum dots, Carbon, 2018, 134, 232-243.

50 R. Yang, X. Guo, L. Jia, et al., Green preparation of carbon dots with mangosteen pulp for the selective detection of $\mathrm{Fe}^{3+}$ ions and cell imaging, Appl. Surf. Sci., 2017, 423, 426432. 